\title{
Comparison of 640-slice Aquilon ONE CT scanner's measured dosimetric parameters with ICRP dose reference levels for head, chest and abdominal CT examinations
}

Samuel Anim-Sampong*, William K. Antwi, Benard Ohene-Botwe and Rosemary S. Boateng

\begin{abstract}
Background: Diagnostic reference levels (DRLs) are optimum range of values or diagnostic standard data for a particular procedure by which other imaging measurements must be compared for purposes of optimizing patient dose and radiation safety in medical imaging. This study aimed to compare measured dosimetric parameters of the latest 640-Slice Aquilion ONE CT scanner with established DRLs for adult and pediatric head, chest and abdominal examinations as a quality assurance test in order to recommend appropriate radiological safety solutions if differences existed.
\end{abstract}

Methods: A prospective and retrospective study design was applied in this work. Data of measured CT dosimetric parameters (patient administered doses) such as dose length product and CT dose index were generated for pediatric head, chest and abdominal examinations using the Aquilion ONE CT scanner. The generated database was compared with established international standard DRLS. SPSS version 16 software was used for data processing and analysis.

Results: The measured volume weighted $C T$ dose index (CTDI $\left.\left.\right|_{\text {vol }}\right)$ and dose length products (DLPs) were generally lower than the ICRP and other internationally recommended DRLs. In particular, the relative deviations from the DRLs were head $\left(C T D I_{\text {vol }}=-57.5 \%, D L P=-33.8 \%, p\right.$-value $\left.=0.001\right)$, chest $\left(C T D I_{\text {vol }}=-94.3 \%\right.$, DLP $=-86.6 \%, p$-value $\left.=0.002\right)$, and abdomen $\left(C T D I_{\text {vol }}=-91.0 \%, D L P=-75.2 \%, p\right.$-value $\left.=0.001\right)$ for pediatric examinations, and head $\left(C T D I_{\text {vol }}=+5\right.$. $5 \%, \mathrm{DLP}=-4.0 \%, p$-value $=0.001)$, chest $\left(C T \mathrm{CI}_{\text {vol }}=-80.3 \%, \mathrm{DLP}=-56.6 \% p\right.$-value $\left.=0.001\right)$, abdomen $\left(C \mathrm{CDI}_{\text {vol }}=-6\right.$. $8 \%, \mathrm{DLP}=-54.7 \%, p$-value $=0.001)$ for adult examinations respectively.

Conclusion: The CTDI $\mathrm{vol}_{\text {val }}$ and DLP administered doses for both pediatric and adult examinations were lower than their respective DRLs except adult head examination where the measured CTDI vol was $5.5 \%$ higher than the standard reference dose. Optimization of practice is needed to reduce dose to head $C T$ examinations when using 640-slice Aquilion ONE CT scanner.

Keywords: Computed tomography, Dose reference level (DRL), Dosimetric parameters, International standards

* Correspondence: tychicus77@gmail.com

Department of Radiography, University of Ghana School of Biomedical and

Allied Health Sciences, P.O. Box KB 143, Korle, Accra, Ghana 


\section{Background}

Computed tomography (CT) is a useful imaging modality incorporating advanced technology to produce high resolution 3-D images $[1,2]$. Due to the associated high radiation dose [3], dose optimization protocols are applied to examination-specific practices to ensure occupational and medical radiological safety and protection [2, 4] in accordance with ALARA and radiation protection principles.

Dose reference levels (DRLs) are established with CT imaging to obtain image quality consistent with the procedure and optimization of radiation as a result of the associated high radiation dose $[5,6]$. They are classified as suggested action levels above which a facility should review its methods and determine if acceptable image quality can be achieved at lower doses, and also considered as investigational levels applied in the identification of unusually high radiation doses [6-9]. The importance of DRLs for assessing existing protocols, developing new and improved protocols, and as a dose optimization tool for promotion of good practices in CT imaging has been affirmed [10-18]. According to the Australian Radiation Protection and Nuclear Safety [19], DRLs provide a common dose metric for the comparison of DRLs between facilities, protocols and modalities, assess dose impacts of newly introduced protocols, and provide compliance with the relevant state and territory regulatory requirements.

In CT imaging, DRLs are set at all levels for each examination and each patient group i.e., adults and children of different sizes [15]. The practical dose quantities set up to monitor $C T$ practice include weighted $\left(C T D I_{w}\right)$ and volume-weighted $\left(C T D I_{v o l}\right)$ CT dose index (CTDI), and dose-length product $(D L P)$ [20]. Accordingly, the importance of establishing facility-specific $C T D I_{v o l}$ and $D L P$ values for each examination type and associated clinical indication [21] has been suggested. The Institute of Physics and Engineering in Medicine (IPEM) [22] has indicated that DRLs should be compared with relevant national DRLs (NDRLs) (if any), and values found higher than the NDRLs investigated and either justified as being clinically necessary or reduced through appropriate modifications in practice to improve occupational and patient protection.

Mathematically, the $C T D I_{v o l}$ quantifies CT scanner output for a specific scan protocol and is introduced to account for the pitch as

$$
\begin{aligned}
C T D I_{v o l} & =\frac{1}{p} C T D I_{w} \\
& =\frac{1}{3 p}\left(C T D I_{100, \text { center }}+2 C T D I_{100, \text { edge }}\right)
\end{aligned}
$$

for a multislice system, and as

$$
\begin{aligned}
C T D I_{v o l} & =\frac{1}{p} C T D I_{w}=\left(\frac{d}{1 / w}\right) C T D I_{w} \\
& =\left(\frac{d}{1 / n \lambda}\right) C T D I_{w}
\end{aligned}
$$

in terms of the travel distance per gantry rotation $(d)$, nominal beam width $(w)$ and number $(n)$ of sections per scan. The corresponding $D L P$ during the complete examination is computed [23] via

$$
\begin{aligned}
D L P & =L\left(C T D I_{v o l}\right)=\frac{L\left(C T D I_{w}\right)}{p} \\
& =\left(\frac{n \lambda}{I}\right) C T D I_{w}=\sum_{i}\left(n C T D I_{w}\right) i \lambda t
\end{aligned}
$$

where $L$ and $I$ are scan length interval and interval of scan length respectively, and serial scan sequence $(i)$, number of slices $(N)$, radiographic exposure $(C)$, total acquisition time $(t)$, and tube current $(i)$ are other scan parameters.

Effective dose is the amount of energy deposited in the irradiated organ and used for evaluating radiation dose, [24] and comparison of stochastic effects. It is evaluated from relative radiation risk to each organ and dose to individual organs, taking into account the absorbed dose received by each irradiated organ and organ's sensitivity [25]. Using the International Commission on Radiological Protection 103 tissue-weighting factors $\left(w_{T}\right)$ and organ dose estimate approach, the effective dose can be calculated via

$$
E_{D}=\sum_{z}\left[\sum_{T} w_{T} H_{T}\right]
$$

where $H_{T}$ is the corresponding absorbed dose to the specified tissue $(T)$ [26], derived from the European Commission guidelines and computed $D L P$ values [27] as

$$
E_{D}={ }^{D} k D L P=k\left(L C T D I_{v o l}\right)=\left(\frac{k L}{p}\right) C T D I_{w}
$$

where $k$ is a data set of tissue-weighting coefficients derived from data sets restricted to the scanned anatomic region.

The 640 multislice scanning volume Aquilion ONE TSX-301A CT scanner was recently installed at the Korle Bu Teaching Hospital (KBTH). The scanner supports simultaneous data acquisition of 320 row detector slices of the whole body scanning using the Selectable Slice-thickness Multi-row Detector (SSMD). However, recent Toshiba-initiated [28] recalls of this scanner upon confirmation of a potential software problem which indicated incorrect setting of $\mathrm{x}$-ray output conditions, and consequently presented reliability challenges of the scan 
results have been reported by the US Food and Drugs Administration (FDA) [29].

Radiation doses associated with the operation of this CT scanner at KBTH have not been characterized by comparing measured doses with internationally accepted DRLs, ascertain any differences, validate measurements, and establish a relationship between the DRLs and measured doses. In particular, this is important for purposes of ensuring occupational and medical radiation safety and protection. The absence of such studies presents obvious challenges to safety. Hence in accordance with IPEM [22] indications of the importance for each $\mathrm{CT}$ facility to establish local DRLs (LDRLs) or typical CTDI $I_{\text {vol }}$ and DLP) dose levels for each type of examination and associated clinical indication, this study was performed to measure dosimetric parameters for $\mathrm{CT}$ examinations of the head, chest and abdominal anatomic regions and compare with internationally accepted DRLs. Any observed differences could then be investigated and justified as being clinically necessary or reduced via approved methods such as appropriate changes in clinical practice in order to improve medical radiation protection. Consequently, practicing radiographers and radiologists at this facility will be enabled to adapt good practices to patient dose optimization with respect to the DRLs.

\section{Methods}

A retrospective and prospective design was adopted to obtain data from previous and current examinations at the time of the study. The population consisted of 350 pediatric and adult patients referred for CT examinations of the head, abdomen and chest regions at the study site. Data acquisition was made from automatically generated $C T D I_{\text {vol }}$ and $D L P$ values for each patient scan based on radiographer selected parameters of the 640 multislice Aquilion ONE CT TSX-301A scanner which stores parametric scan data. Automatic dose reduction was ensured by integration of Toshiba's Adaptive Iterative Dose Reduction 3D (AIDR 3D) iterative reconstruction technology into the imaging chain. Images (in 3-D) of the head, chest and abdomen body regions of referred patients were acquired in volume and helical format. The LDRLs $\left(C T D I_{\text {vol }}\right.$ and $D L P$ values) were then established by retrieval of the stored data including the standardized DRLs on the CT scanner. In this study, the ICRP DRLs were chosen as the main reference by which the measured DRLs were compared and deviations determined. Comparisons with DRLs specified by the American College of Radiology, American Association of Physicists, European Commission, and those used in Sweden and the United Kingdom were also made.
Paired sample T-test was used to compare the DRLs and the patient measured doses for each examination. A two-tail statistical significance with a $p<0.05$ value was determined.

\section{Ethics}

The Ethical and Protocol Review Committee of the University of Ghana School of Biomedical and Allied Health Sciences, and management of the Radiology Department of KBTH approved the study. For the prospective study, the consent of adult patients was directly sought while parental consent was obtained from parents/guardians of pediatric patients. For the retrospective study, consent to access patients' data for the study was also approved by the management of KBTH. Demographic and scan data of patients referred for head, abdomen and chest CT examinations at the study site during the study period were included in the study. These CT examinations were selected because they constitute the most common or frequently requested examinations at the Centre. Conversely, patients' data obtained before and after the study period were excluded from the study. Additionally, data obtained from CT examinations other than the head, chest and abdomen regions were excluded from the study.

\section{Results}

The age and gender demographics of the participants are presented in Table 1. The ages of the pediatric patients ranged from less than 5 years to 10 years. The number of head CT scans performed for the 6-10 year group was marginally higher $(n=37,51.4 \%)$ than those in the less than 5 year group $(n=35,48.6 \%)$. The same frequencies $(n=2,50 \%)$ were however obtained for both pediatric age groups referred for chest and abdomen CT examinations. There were more pediatric $\mathrm{CT}$ head referrals $(n=72,90 \%)$ compared to $5 \%(n=4)$ each for chest and abdomen CT examinations.

The age range of the adults was 18 years to 100 years. Referrals for CT head and chest examinations were higher among the 36-59 age group (head: $n=130,46.8 \%, n=20,43.5 \%)$ compared to the 60 100 years (head: $n=79,28.4 \%$; chest: $n=15,32.6 \%$ ). The number of CT referrals for abdominal; examination was the same $(n=33,38.4 \%)$ for both age groups however. The least referrals for all three modalities was registered among the 18-35 year group (head: $n=69,24.8 \%$; chest: $n=6,13 \%$; abdomen: $n=20,23.3 \%)$.

More male pediatric CT head $(n=42,58.3 \%)$ and abdominal $(n=3,75 \%)$ examinations were studied compared to $41.7 \%(n=30)$ and $25 \%(n=1)$ for females. On the contrary, more $(n=3,75 \%)$ peditric chest examinations were registered for females than for males $(n=1$, 
Table 1 Age and gender demographics

\begin{tabular}{|c|c|c|c|c|c|c|c|}
\hline \multirow{3}{*}{$\begin{array}{l}\text { Patient } \\
\text { category }\end{array}$} & \multirow{3}{*}{$\begin{array}{l}\text { Demographic } \\
\text { variable }\end{array}$} & \multicolumn{6}{|c|}{ Type of CT examination } \\
\hline & & \multicolumn{2}{|l|}{ Head } & \multicolumn{2}{|c|}{ Chest } & \multicolumn{2}{|c|}{ Abdomen } \\
\hline & & No. & Percent, $\%$ & No. & Percent, $\%$ & No. & Percent, $\%$ \\
\hline \multicolumn{8}{|c|}{ Age demographics } \\
\hline \multirow[t]{3}{*}{ Pediatrics } & $\leq 5$ years & 35 & 48.6 & 2 & 50.0 & 2 & 50.0 \\
\hline & $6-10$ years & 37 & 51.4 & 2 & 50.0 & 2 & 50.0 \\
\hline & Total & 72 & 100.0 & 4 & 100.0 & 4 & 100.0 \\
\hline \multirow[t]{4}{*}{ Adults } & $18-35$ years & 69 & 24.8 & 6 & 13.0 & 20 & 23.3 \\
\hline & $36-59$ years & 130 & 46.8 & 20 & 43.5 & 33 & 38.4 \\
\hline & $60-100$ years & 79 & 28.4 & 15 & 32.6 & 33 & 38.4 \\
\hline & Total & 278 & 100.0 & 41 & 100.0 & 86 & 100.0 \\
\hline \multicolumn{8}{|c|}{ Gender demographics } \\
\hline \multirow[t]{3}{*}{ Pediatrics } & Male & 42 & 58.3 & 1 & 25.0 & 3 & 75.0 \\
\hline & Female & 30 & 41.7 & 3 & 75.0 & 1 & 25.0 \\
\hline & Total & 72 & 100.0 & 4 & 100.0 & 4 & 100.0 \\
\hline \multirow[t]{3}{*}{ Adults } & Male & 150 & 54 & 26 & 63.4 & 42 & 48.8 \\
\hline & Female & 128 & 46 & 15 & 36.6 & 44 & 51.2 \\
\hline & Total & 278 & 100.0 & 41 & 100.0 & 86 & 100.0 \\
\hline
\end{tabular}

Source: field data, (2014)

$25 \%)$. The tally of the adult CT scan reports showed more males for head $(n=150,54 \%)$ and chest $(n=26$, $56.5 \%)$ scans compared to $46 \%(n=128)$ and $32.6 \%(n$ =15) for females respectively. More females $(n=44$, $51.2 \%)$ however, registered for abdominal scans than males $(n=42,48.8 \%)$.

\section{Administration of Intravenous Contrast Media}

Intravenous (IV) contrast media was administered to some of the patients (Fig. 1). All (100\%) the abdominal CT referrals for pediatric patients were administered with IV contrast media while administrations for chest and head referrals were $75 \%(n=3)$ and $52.8 \%(n=38)$ were respectively. Overall, $51.3 \%(n=41)$ of pediatrics received IV contrast administration. The clinical histories associated with these examinations were mainly hydrocephalus, seizures, and trauma. Similarly, $40.7 \%$ $(n=61)$ of adult head CT referral cases received IV contrast media. As observed for the pediatric patients, higher rates of IV contrast administrations were recorded for adult CT referrals for chest ( $n=38,92.7 \%)$ and abdomen ( $n=77,89.5 \%)$. The registered clinical histories for the adult referrals were cerebrovascular accident (CVA), intracranial space occupying lesion

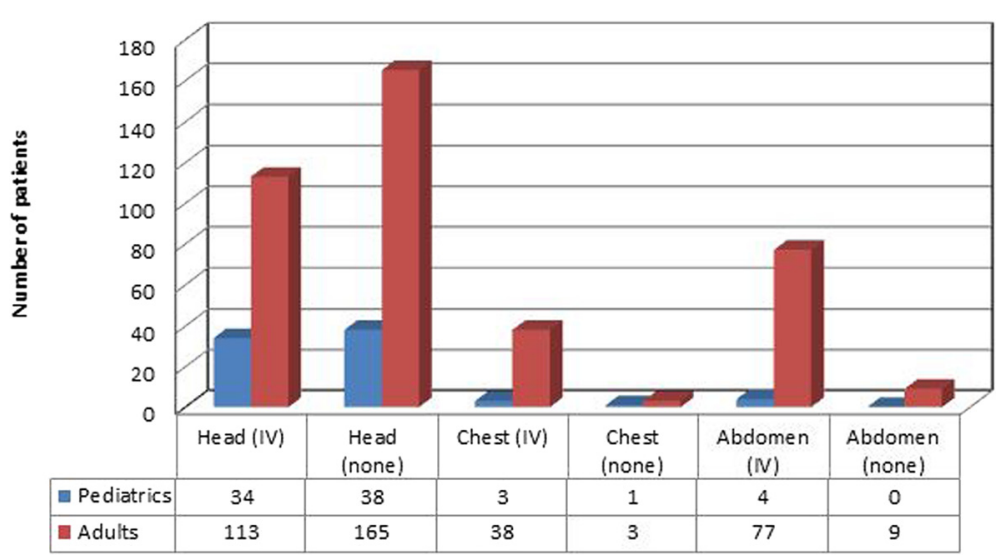

Fig. 1 IV contrast administration to paediatric and adult CT examination 
(ICSOL) and trauma (head CT), ccancer of the lung and pulmonary $\mathrm{TB}$ (chest $\mathrm{CT}$ ), and abdominal pains (abdomen CT).

\section{Dosimetry \\ Head, chest and abdominal examinations}

The results of the measured doses compared with the ICRP DRLs for CT head, chest and abdominal scans are presented in Table 2. The means of the measured $C T D I_{\text {vol }}$ values for pediatric and adult doses were $23.87 \pm$ $10.18 \mathrm{mGy}$ and $63.29 \pm 8.94 \mathrm{mGy}$ respectively. Comparatively, the measured doses were lower than the ICRP DRL value of $56.11 \pm 12.843$ for pediatrics, and higher than $60.00 \mathrm{mGy}$ for adult the ICRP DRLStatistically, a $p$-value of $0.001<0.005$ with a 0.95 confidence interval was estimated which established a significant difference between the measured doses and the DRLs. The measured DLP doses for pediatric and adult CT head scans were 406.59 $\pm 71.121 \mathrm{mGy}$, and 1008.26 $\pm 266.730 \mathrm{mGy}$. Comparatively, the measured DLPs were lower than the ICRP DRLs and calculated $p$-values $(<0.005)$ established significant differences between them. Relative deviations of the measured values (LDRL) from the reference (DRL) values were calculated via eqn. (6)

$$
\text { Rel. } \quad \text { dev. }=\left(\frac{L D R L-D R L_{r e f}}{D R L_{r e f}}\right) x 100 \%
$$

The measured chest $C T D I_{\text {vol }}$ values (pediatrics: 1.70 \pm 10.18 mGy; adults: $5.92 \pm 3.14$ mGy) were very significantly lower than the ICRP DRLs (30.00 \pm $0.00 \mathrm{mGy}$ ) for both pediatrics and adults withcalculated $p$-values of $0.001-0.002 \quad(<0.005$ at 0.95 confidence interval). Similarly, the measured DLPS (pediatrics: $67.20 \pm 69.54 \mathrm{mGy}$; adults: $282.14 \pm$ $159.12 \mathrm{mGy}$ ) were very significantly lower than the ICRP DRLs (pediatrics: $500.00 \pm 115.470$; adults: $650 \pm$ $0.00 \mathrm{mGy}$ ). The estimated $p$-value was $0.001<0.005$.

The same trend was observed for the abdominal CT examinations for both categories of patients. These results indicated the measured dosimetric parameters were much lower compared to the ICRP DRLs. In particular, the relative deviations of the measured $C T D I_{v o l}$ and DLPs from the ICRP DRLs were $91.0 \%(2.70 \pm$ 1.16 mGy vrs. $30.00 \pm 5.77 \mathrm{mGy})$ and $75.2 \%$ (144.50 \pm 93.57 vrs. $375.00 \pm 144.34$ mGy) respectively for pediatrics, and $80.6 \%(6.79 \pm 3.02$ vrs. $35.00 \pm 0.00 \mathrm{mGy})$ and $54.7 \% \quad(353.48 \pm 178.394$ mGy $\quad$ vrs.780.00 \pm $0.00 \mathrm{mGy})$ ) for adults respectively. For both categories of patients, an estimated p-value of $0.001<0.005$ was statistically established a significant difference the measured and standard DRLs.

Using the ICRP recommended tissue weighting coefficients, the computed effective doses from the CTDI ${ }_{\text {vob }}$ $C T D I_{w}$ and DLPs for each measured DLP for the three body regions are shown in Table 3 . The calculated effective doses for both categories of patients were lower than the ICRP recommended values. The least deviation of $4.0 \%$ was registered for adult head CT effective dose (10.8 mGy) compared to the ICRP value (10.50 mGy), while the highest deviation (86.6\%) of effective dose (8.06 mGy) relative to the ICRP value (60.0 mGy) was observed for pediatric chest. Effective doses for abdominal CT examinations were lower in pediatrics (11.16 mGy, $75.2 \%$ rel. deviation) than adults (42.42 mGy, 54.4 \% rel. dev.).

\section{Discussion}

A study conducted to assess and compare doses a new 640-Slice Aquilion ONE CT scanner with established DRLs for adult and pediatric patients undergoing CT examinations of head, chest and abdominal regions was successfully realized. For both pediatric and adult patients, the lowest frequencies of CT referrals were recorded for chest $(n=45,9.3 \%)$ and $(n=90,8.6 \%)$ for abdomen examinations compared to over $72 \%(n=350)$ for head CT examination. The findings are agreeable with the literature which indicates increasing numbers of head CT scans [4]. More adults presented for head CT scan than pediatrics as this procedure is usually not requested for pediatric patients unless it is very necessary. This is consistent with concerns about cancer risks from exposure to ionizing radiation arising from

Table 2 ICRP DRLS and measured dosimetric parameters for pediatric and adult CT head, chest and abdominal scans

\begin{tabular}{|c|c|c|c|c|c|c|c|c|}
\hline \multirow{2}{*}{$\begin{array}{l}\text { Body } \\
\text { Region }\end{array}$} & \multirow{2}{*}{$\begin{array}{l}\text { Patient } \\
\text { category }\end{array}$} & \multicolumn{3}{|c|}{$\mathrm{CTDI}_{\mathrm{vol}} \pm \mathrm{s} . \mathrm{d}(\mathrm{mGy})$} & \multicolumn{3}{|c|}{$\mathrm{DLP} \pm$ s.d. $(\mathrm{mG} / \mathrm{cm})$} & \multirow[t]{2}{*}{$p$-value } \\
\hline & & ICRP DRL & Measured & Rel. dev. \% & ICRP DRL & Measured & Rel. dev. \% & \\
\hline \multirow[t]{2}{*}{ Head } & Pediatric & 56.11 & $23.87 \pm 10.18$ & -57.5 & 614.58 & $406.59 \pm 10.18$ & -33.8 & 0.001 \\
\hline & Adult & 60.00 & $63.29 \pm 10.18$ & 5.5 & 1050.00 & $1008.26 \pm 10.18$ & -4.0 & 0.001 \\
\hline \multirow[t]{2}{*}{ Chest } & Pediatric & 30.00 & $1.70 \pm 10.18$ & -94.3 & 500.00 & $67.20 \pm 10.18$ & -86.6 & 0.002 \\
\hline & Adult & 30.00 & $5.92 \pm 10.18$ & -80.3 & 650.00 & $282.14 \pm 10.18$ & -56.6 & 0.001 \\
\hline \multirow[t]{2}{*}{ Abdomen } & Pediatric & 30.00 & $2.70 \pm 10.18$ & -90.0 & 375.00 & $93.00 \pm 10.18$ & -75.2 & 0.001 \\
\hline & Adult & 35.00 & $6.79 \pm 10.18$ & -80.6 & 780.00 & $353.48 \pm 10.18$ & -54.7 & 0.001 \\
\hline
\end{tabular}


Table 3 Effective dose

\begin{tabular}{llcccc}
\hline $\begin{array}{l}\text { Body } \\
\text { region }\end{array}$ & $\begin{array}{l}\text { Patient } \\
\text { category }\end{array}$ & $\begin{array}{l}\text { Weighting } \\
\text { factor } W_{T}\end{array}$ & \multicolumn{4}{l}{ Effective dose $E_{D}$ (mGy) } & \\
\cline { 5 - 6 } Head & Pediatric & 0.01 & 6.15 & 4.07 & -33.8 \\
& Adult & 0.01 & 10.50 & 10.08 & -4.0 \\
\multirow{2}{*}{ Chest } & Pediatric & 0.12 & 60.00 & 8.06 & -86.6 \\
& Adult & 0.12 & 78.00 & 33.86 & -56.6 \\
Abdomen & Pediatric & 0.12 & 45.00 & 11.16 & -75.2 \\
& Adult & 0.12 & 93.60 & 42.42 & -54.4 \\
\hline
\end{tabular}

Rel. dev. relative deviation from reference DRLs, s.d. standard deviation

increased use of $\mathrm{CT}$ in pediatrics. In particular, it has been reported that the increased use of $\mathrm{CT}$ in pediatrics, combined with the wide variability in radiation doses resulted in administration of higher doses to many children and that dose-reduction strategies targeted to the highest quartile of doses could dramatically reduce the number of radiation-induced cancers [30]. The observed lower pediatric referrals for head CT scan in this study is also confirmed by a study [31] which identified the lack of clinical significant findings associated with the vast majority of pediatric head CT scans and recommended reductions in the frequency of $\mathrm{CT}$ referrals as well as implementation of interdisciplinary measures in advocating for decreased and unnecessary CT imaging of pediatrics.

More males $(n=264,54.4 \%)$ CT examinations were studied compared to females $(n=221,45.6 \%)$ for all the three examinations. The higher male ratio could be attributed to the fact that many males suffered more trauma cases than females as evidenced via the noted clinical trauma histories in the various examinations.

\section{Contrast examination}

Intravenous contrast (IV) contrast media are administered in radiographic examinations to make the anatomy of interest more visible. The study showed that about $55.5 \%$ of all the CT examinations were administered with contrast media (head: $30.3 \%$; chest: $8.5 \%$ and $16.7 \%$ ) whileall (100 \%) the abdominal CT referrals were IV contrast media-administered for the pediatric examinations. The clinical histories associated with these examinations were mainly hydrocephalus, seizures, and trauma for pediatrics, while cerebrovascular accident, intracranial space occupying lesion (head CT), cancer of the lung and pulmonary tuberculosis (TB) (chest $\mathrm{CT}$ ), and abdominal pains (abdomen $\mathrm{CT}$ ) were registered for adults. These findings agree with the work of Wang and You [32], which revealed clinically important abnormalities such as history of malignancy, focal neurologic deficit in a study of clinical predictors of abnormal findings of head CT non-trauma patients. Contrary to these however, the prediction of abnormalities on head CT as indications of increased risk of lumbar puncture-induced brain herniation history and physical examination findings has been queried [33].

\section{Dosimetry}

The LDRL or measured mean weighted $C T D I_{\text {vol }}$ value of $63.29 \pm 8.94 \mathrm{mGy}$ for adult head CT examination was consistent with the ICRP [34] and European Commission [35] DRL values 60 mGy with a $5.5 \%$ deviation. In another study [5], different DRLs were reportedly used by imaging sites which demonstrated abrupt transition at $60 \mathrm{mGy}$, with increasingly higher values in order to produce the desired image quality. A new $C T D I_{\text {vol }}$ value of $75 \mathrm{mGy}$ was therefore set for head CT examination [16]. Comparatively, the measured adult head $C T D I_{\text {vol }}$ value of $63.29 \pm 8.94 \mathrm{mGy}$ reported in this work is lower than the ACR-AAPM and EUR 16262 DRLs as well as the published NDRLs for the United Kingdom 2003 (65$100 \mathrm{mGy}$ ), Switzerland 2010 (65 mGy), and Sweden 2002 (75 mGy) respectively [36-38]. Generally, the relative deviations of the LDRLs were least with respect to the ACR values. Taking cognizance of the fact that the precise rationale of established DRLs is to provide a benchmark for comparison, and not to define a maximum or minimum dose limit as stated by the American College of Radiology [6], the measured LDRL, though higher by $5.5 \%$ with respect to the ICRP DRL ((60.0 mGy) is however $15.6 \%$ lower than the ACR's new $C T D I_{\text {vol }}$ value of $75 \mathrm{mGy}$ for head CT. Since ACRs new values do not impact negatively on patient radiological safety but are suitable for higher image quality, then the measured LDRLs in this study is within limits of patient radiological safety and would thus not induce any biological damage to the adult patients undergoing head CT examinations using this CT scanner. On the basis of this, it is expected that dose optimization strategies, as well as audit and practice review for promoting improvements in patient medical protection will be required and implemented as recommended by the Institute of Physics and Engineering in Medicine [39].

The results of the study further showed that the $C T D I_{\text {vol }}$ measured doses for pediatric head, as well as pediatric and adult chest and abdominal CT scans were much lower than the ICRP, ACR-AAPM and EUR DRLs. The relative deviations ranged from $57.5 \%$ to $94.3 \%$ in pediatrics, and $5.5 \%$ to $80.6 \%$ in adults. These results are in agreement with another study [4] which reported radiation doses from adult CT procedures $90 \%$ below the $C T D I_{v o l}$ reference levels. In terms of patient safety, the administered or measured doses generally did not exceed internationally accepted and recommended DRLS. This means that patients were not inadvertently exposed to radiation. Also the findings of the study are suggestive the scanner was safely operated within its 
operating limits and conditions (OLCs) to ensure patient and occupational safety.

The findings of this study showed that all the measured mean $D L P$ values for head, chest and abdomen CT examinations for both pediatrics and adults were lower than their respective ICRP DRLs. This is indicative that all administered doses were consistent with ALARA leading to dose optimization. It was reported [4] that the measured $D L P$ exposures for adult CT procedures were below the $D L P$ reference levels by $62 \%$ and thus supports the findings of this study which recorded deviations in the range of 4.0 to $56.6 \%$. Generally, the deviations of the measured dosimetric parameters from the standard ICRP DRLs are consistent with published literature (Tsalafoutas et al., 2012=40). However, the degree of deviation depends on the type of CT scanner used, the imaging parameters and other physical operating conditions. In particular, the reported small deviations [40] were based on calculating patient effective dose via a comprehensive method in which 3 different CT scanners were used in $30 \mathrm{CT}$ examinations of the chest, chest-abdomen-pelvis, and abdomen-pelvis regions. Statistically, $p$-values in the range of $0.001-0.002$ $<0.005$ with a $0.95 \mathrm{CI}$ established significant differences between the measured dosimetric parameters and the ICRP reference doses.

Fundamentally, effective doses reflect the choice of scanning protocol (radiographic technique factors) such as such as mAs values, scan length, and pitch used in a given CT examination. In this study, the calculated effective doses from the measured DLPS were lower than the ICRP reference values, but higher than those reported elsewhere in the literature, particularly for adults. Effective computed doses of $1.3 \mathrm{mGy}$ have been reported for head examinations [24], 5.4 mGy for chest examinations [41], and 9 mGy for abdominal examinations [42], using different approaches.

It has been reported that pediatric doses are currently lower than previously reported. Using dose reduction strategies, effective dose reductions from $9 \mathrm{mGy}$ to $1.5 \mathrm{mGy}$ for pediatrics weighing up to $10 \mathrm{~kg}$ for chest examinations and a corresponding reduction for abdominal CT examination from $7 \mathrm{mGy}$ to $2 \mathrm{mGv}$ have been indicated [42]. Comparatively the results of this study do not agree with this reported work [42]. The reasons for the differences could be attributed to the different imaging protocols and non-availability of useful diagnostic information at the time of the measurements. However, in accordance with dose optimization principles and medical radiation protection and safety principles, this study identifies with the fact that the increased radiosensitivity of children and the increasing utilization of CT require significant dose reduction strategies for pediatrics without apparent loss of diagnostic information which is consistent with the literature [43].

\section{Conclusions}

A comparative study of measured dosimetric parameters with ICRP and other internally recognized DRLs for pediatric head, chest and abdominal examinations has been done using the 640-Slice Aquilion ONE CT scanner facility in a tertiary referral hospital. The facility does not display any facility-specific DRLs on its monitors. Hence for purposes of this study, the well utilized and reliable ICRP 103 DRLs was used as the main reference DRLs. With the exception of CTDI $I_{\text {vol }}$ values measured for adult head CT, all the measured values for head, chest and abdominal CT procedures, were generally consistent with the literature and lower than the standard ICRP, EUR 16262, ACR, AAPM and other NDRLs (Sweden, Switzerland, United Kingdom). Statistically, significant differences between the measured $C T D I_{\text {vol }}$ and DLP values, and the corresponding ICRP DRL values were established. The effective doses were lower in pediatrics than in adults which reflected the choice of scanning protocols adopted for the CT examinations.

In accordance with dose optimization, medical radiation protection and safety principles, this study identifies with the fact that the increased radio-sensitivity and increased utilization of $\mathrm{CT}$ require implementation or review of significant dose reduction strategies for optimization of practice using this scanner for CT head examinations. This has to be done without apparent loss of diagnostic information in accordance with ALARA.

\section{Abbreviations}

ALARA, as low as reasonably achievable; CT, computed tomography; DRLs, diagnostic reference levels

\section{Acknowledgement}

The authorship of this paper is grateful to the management of the Radiology Department of KBTH for the use of its Aquilion ONE CT scanner for this study

\section{Availability of data and materials}

Data for this study has been duly presented in the text and tables of this manuscript.

\section{Authors' contributions \\ Authors SAS, BOB, WKA and RSB designed the study, performed the statistical analysis, wrote the protocol, and wrote the first draft of the manuscript. All authors read and approved the final manuscript.}

\section{Competing interest}

The authors declare that they have no competing interests.

\section{Ethics approval and consent to participate}

The Ethical and Protocol Review Committee of the University of Ghana School of Biomedical and Allied Health Sciences approved the study. For the prospective study, patients' consents were sought for this study. Parental consent was obtained from parents/guardians in cases where the participant was under 18 years. For the retrospective study, consent to access patients' data for the study was also approved by the management of the Radiology Department of KBTH. Therefore, the need for consent was waived by the IRB for the data collected retrospectively. These approvals included consents to publish this work. 
Received: 9 June 2016 Accepted: 26 July 2016 Published online: 02 August 2016

\section{References}

1. Kim S, Song H, Samei E, Yin FF, Yoshizumi TF. Computed tomography dose index and dose length product for cone-beam CT: Monte Carlo simulations of a commercial system. J Appl Clin Med Phys. 2011;12(2):256-69.

2. Ogbole Gl. Radiation dose in Pediatric computed tomography: Risks and benefits. Ibadan Ann Postgrad Med. 2010;8(2):118-21.

3. Brenner J, Elliston D, Hall J, Berdon E. Estimated risks of radiation-induced fatal cancer from pediatric CT. Am J Rev. 2001;176:289-96.

4. Wambani JS, Korir GK, Onditi EG, Korir IK. A survey of computed tomography imaging techniques and patient dose in Kenya. East Afr Med J. 2010;87(10):400-6.

5. McCollough $\mathrm{CH}$, Leng S, Yu L, Cody DD, Bonne JM, McNitt-Gray MF. CT Dose Index and Patient dose: they are not the same thing. Radiol J. 2011; 256:311-6.

6. American College of Radiology-American Association of Physicists in Medicine ACR-AAPM Practice guideline for diagnostic reference levels and achievable doses in medical x-ray imaging. 2013. http://www.acr.org/ guidelines.

7. Gray JE, Archer BR, Butler PF, Hobbs BB, Mettler FA, Pizzutiello RJ, Yaffe MJ. Reference values for diagnostic radiology: application and impact. Radiol J. 2005:235:354-8

8. Hart, D, Hillier, MC, Wall, BF. Doses to patients from radiographic and fluoroscopic x-ray imaging procedures in the UK-2005. 2007 Review. http:// www.hpa.org.uk

9. National Council on Radiation Protection and Measurement Diagnostic Reference levels and achievable doses in medical and dental imaging: Recommendations for the United States. Bethesda, MD. NCRP Report No. 172. 2012.

10. Shrimpton PC, Hillier MC, Lewis MA, Dunn M. National survey of doses from CT in the UK: 2003. Br J Radiol. 2006;79:968-80.

11. Tsapaki V, Aldrich JE, Sharma R, Staniszewska MA, Kansaachinda A, Rehani $M$, Prokop $M$. Dose reduction in $C T$ while maintaining diagnostic confidence: diagnostic reference levels at routine head, chest, and abdominal CT: IAEA-coordinated research project. Radiol J. 2006;240:828-34.

12. Muhogora WE, Ahmed NA, Beganovic A, Benider A, Graj-Bjelac O, Gershan $\checkmark$, Rehani MM. Patient doses in CT examinations in 18 countries: initial results from International Atomic Energy Agency projects. Radiat Prot Dosimetry. 2009:136:118-26.

13. Dosimetry Working Party of the Institute of Physical Sciences in Medicine. National protocol for patient dose measurements in diagnostic radiology. Chilton: National Radiological Protection Board; 1992.

14. International Commission on Radiological Protection. Radiological protection and safety in medicine. Report No 73. Ottawa: International Commission on Radiological Protection; 1996.

15. International Commission on Radiological Protection Publication 87 (2001) Managing Patient Dose in Computed Tomography. 2001. http://w3.tue.nl/ fileadmin/sbd/Documenten/Leergang/BSM/ICRP87_patient_dose_in_CT.

16. American College of Radiology. ACR practice guideline for diagnostic reference levels in medical $x$-ray imaging. In: American College of Radiology Practice guidelines and technical standards. Reston: American College of Radiology; 2008. p. 799-804.

17. International Atomic Energy Agency. Technical meeting on patient dose monitoring and the use of diagnostic reference levels for the optimization of protection in medical imaging. https://rpop.iaea.org/rpop/rpop/Content/ News/6-tm-patient-dose-monitoring.htm.

18. European Commission. European guidelines on quality criteria for computed tomography. Report No EUR 16262 EN. Luxembourg: European Commission \& the Office for Official Publications of the European Communities; 2000.

19. Australian Radiation Protection and Nuclear Safety Agency. ARPANSA RPS 14 Code of practice for radiation protection in the medical applications of ionizing radiation. Yallambie: Australian Radiation Protection \& Nuclear Safety Agency; 2008

20. Bongartz G, Golding S, Jurik A, Leonardi M, Merteen E, Geleijns J, Tosi G. European Guidelines for multislice computed tomography. Luxembourg: European Commission \& the Office for Official Publications of the European Communities; 2004.
21. Morin RL, Gerber TC, McCollough CH. Radiation dose in computed tomography of the heart. Circulation. 2003:107:917-22.

22. Institute of Physics and Engineering in Medicine, IPEM. Guidance on the establishment and use of diagnostic reference levels for medical $x$-ray examinations. IPEM report 88. 2004.

23. Hatziioannou K, Papanastassiou E, Delichas M, Bousbouras P. A contribution to the establishment of diagnostic reference levels in CT. Br J Radiol. 2003; 76:541-5.

24. Huda W, Chamberlain CC, Rosenbaum AE, Garrisi W. Radiation doses to infants and adults undergoing head CT examinations. Med Phys J. 2001;28: 393-9.

25. Olarinoye $I O$, Sharifat I. A protocol setting dose reference level for medical radiography in Nigeria. Bayero J Pure Appl Sci. 2010;3(1):138-41.

26. International Commission on Radiological Protection. The 2007 recommendations of the International Commission on Radiological Protection: International Commission on Radiological Protection Publication 103. Ann ICRP. 2007;37(2-4):326-30.

27. Deak D, Smal Y, Kalender A. Multisection CT protocol: sex and age specific conversion factors used to determine effective dose from dose-length product. Med Phys J Radiol. 2010;257(1):284-94.

28. Toshiba Medical Systems Europe (2014). Aquilion ONE ${ }^{\mathrm{TM}}$ : World's first dynamic volume CT system. 2014. http://www.toshiba-medical.eu.CT/ Systems/Aquilion ONE.

29. Food and Drug Administration. What's Nationwide Evaluation of X-ray Trends. 2012. http://www.crcpd.org/Pubs.

30. Miglioretti D, Johnson E, Williams A, Greenlee T, Weinmann S, Leif I, SmithBindman R. The use of computed tomography in pediatrics and the associated radiation exposure and estimated cancer risk. JAMA Pediatr. 2013;167(8):700-7.

31. Owlia M, Yu L, Deible C, Hughes MA, Jovin F, Bump GM. Head CT scan overuse in frequently admitted medical patients. Am J Med. 2014;127(5): 406-10.

32. Wang $X$, You JJ. Head CT for non- trauma patients in the emergency department: clinical predictors of abnormal findings. Radiol J. 2013;266(3): 783-90.

33. O'Laughlin KN, Jerome R, Hoffman JR, Go S, Gabayan GZ, Iqbal E, Merchant G, Mower WR. Non-concordance between clinical and head CT findings: The specter of over diagnosis. Emerg Med Int. 2013;2013:7. Article ID 314948

34. International Commission on Radiological Protection. Diagnostic reference levels in medical imaging: Review and additional advice. 2000. http://www. icrp.org/docs/DRL-for-web.

35. European Commission. European guidelines on radiation protection in dental radiology. Luxembourg: Office for Official Publications of the European Communities; 2004.

36. Shrimpton PC, Hillier MC, Lewis MA, Dunn M. National survey of doses from CT in the UK. Br J Radiol. 2003;79:968-80.

37. Treier R, Aroua A, Verdun FR, Samara E, Stuessi A, Trueb PR. Patient doses in CT examinations in Switzerland: Implementation of national diagnostic reference levels. Radiat Prot Dosimetry. 2010;142:244-54.

38. Swedish Radiation Protection Authority. Regulations and general advice on diagnostic standard doses and reference levels within medical $x$-ray diagnostics. Stockholm: Swedish Radiation Protection Authority; 2002.

39. Institute of Physics and Engineering in Medicine. Recommended standards for the routine performance testing of diagnostic $x$-ray imaging systems. IPEM report 91. 2005.

40. Tsalafoutas IA, Thalassinou S, Efstathopoulos EP. A comprehensive method for calculating patient effective dose and other dosimetric quantities from CT dicom images. Am J Rev. 2012;199:133-41.

41. Huda W, Scalzetti EM, Roskopf M. Effective doses to patients undergoing thoracic computed tomography examinations. Med Phys J. 2000:27:838-44.

42. Ware DE, Huda W, Mergo PJ, Litwiller AL. Radiation effective doses to patients undergoing abdominal CT examinations. Radiol J. 1999;210:645-50.

43. Linton OW, Mettler FA. National Council on Radiation Protection and Measurements. National conference on dose reduction in $\mathrm{CT}$, with an emphasis on pediatric patients. Am J Rev. 2003:181:321-9. 